\title{
Dengue encephalitis in a child
}

\section{J Kudalugoda Arachchi ${ }^{1}$, R Ajanthan ${ }^{2}$, S P Lamabadusuriya ${ }^{3}$ and S Gunasena ${ }^{4}$}

(Index words: Decorticate rigidity, seizures)

\section{Introduction}

Sri Lanka is endemic for dengue infection, and over 3000 cases are reported [1]. Dengue predominantly affects children, presenting as classical dengue, a self-limiting but more severe form of febrile illness. It can be complicated in a few, causing dengue haemorrhagic fever (DHF) and dengue shock syndrome (DSS). Neurological manifestations associated with dengue fever have been reported but are rare $[2,3]$.

1,2, 3 Faculty of Medicine, University of Colombo; ${ }^{4}$ Virologist, Medical Research Institute, Colombo.

Correspondence: JKA, Department of Paediatrics, Faculty of Medicine, Kynsey Road, Colombo 8, Tel: +94 11 695300, e-mail: nwanige@ hotmail.com (Competing interests: none declared) Received 12 February and revised version accepted 8 October 2003. 


\section{Case report}

A 9-year old girl was admitted to Lady Ridgeway Hospital complaining of fever for four days and rightsided abdominal pain for three days. She had been vaccinated against Japanese encephalitis infection. On admission she was flushed, with a positive Hess test. Her pulse rate was 96 per min and blood pressure 100/60 $\mathrm{mmHg}$. The extremities were warm. The liver was tender, enlarged to $2 \mathrm{~cm}$ below the right costal margin.

Next day she developed three bouts of coffee ground vomitus. A diagnosis of dengue haemorrhagic fever was made. On the fifth day of illness, rapid deterioration of her sensorium occurred from nonsensical speech to decorticate rigidity (Glasgow coma scale of 5/15), despite a stable cardiovascular status. She also had generalised seizures and was transferred to the intensive care unit (ICU).

At the ICU, the peripheral circulation deteriorated, the packed cell volume increased to $48 \%$ and the platelet count dropped to $25 \times 10^{9} / \mathrm{L}$. Serum transaminases (SGPT > $110 \mathrm{iu} / \mathrm{L}, \mathrm{SGOT}>105 \mathrm{iu} / \mathrm{L})$ and prothrombin time (test 40s, control 14s) were elevated. Random blood glucose and serum electrolytes remained normal throughout.

The electroencephalogram (EEG) showed marked slowing of background activity. Computed tomography (CT) scan of the brain showed cerebral oedema but no intracranial haemorrhage. Although the cerebrospinal fluid (CSF) report showed no cells and had a protein content of $28 \mathrm{mg} / \mathrm{dL}$, her altered consciousness associated with fever and generalised convulsions was compatible with encephalitis [2]. She was treated with intravenous mannitol, aciclovir and platelet and plasma infusions. She did not require ventilator support.

The serum taken on 10th day of illness was positive for dengue IgM antibodies. Her IgG antibody titre on the same day was < 20 HAI units for dengue virus subtype 2 which rose to $320 \mathrm{HAI}$ units during the convalescent period (day 24), confirming a primary dengue infection of subtype 2. Her serum IgM was negative for Japanese encephalitis, herpes simplex, and mumps. The CSF IgM was negative for Japanese encephalitis, dengue, herpes simplex and mumps. The nasopharyngeal aspirate was negative for influenza antigens.

She remained in decorticate rigidity for a further 2 days. Thereafter, her level of consciousness gradually improved over the next 5 weeks. She was discharged from the hospital 37 days after the onset of illness with no residual motor or sensory weakness and near normal cognitive functions.

\section{Discussion}

Although neurological manifestations associated with dengue infection have received little attention in the past, there have been recently a number of cases reported
$[2,3]$. The incidence has been reported as $1 \%$ of all dengue admissions in a study conducted in Vietnam [2]. Out of the 378 cases of suspected central nervous system (CNS) infection reported in this study, 16 cases $(4.2 \%)$ were due to dengue infection.

Such manifestations have been attributed to CNS invasion by the virus, associated complications or concomitant infection of the CNS by other arboviruses [2]. The complications implicated are hypotension, microvascular or frank intracranial haemorrhage, and encephalopathy due to cerebral oedema, hyponatraemia and associated hepatic failure [2,3,4]. Clinical features of neurological manifestations reported include altered consciousness (Glasgow coma scale < 14), severe headache, neck stiffness, focal neurological signs, generalised convulsions, tense fontanelle, mono- or polyneuropathies and transverse myelitis [2-5].

Neurological manifestations are commonly associated with dengue type 3 (DEN-3) infection [2]. Detection of IgM antibodies in the CSF is diagnostic of dengue CNS infection [7]. This is done using an ELISA technique. The CSF may show a pleocytosis with increase in protein level, but it may be acellular.

The outcome of patients with dengue encephalitis admitted to the Centre for Tropical Diseases in Southern Vietnam had a median coma recovery time of 3.5 days (range 1-45). In this group of 16 patients no deaths were reported but six had neurological sequelae on discharge (2). In Sri Lanka since dengue fever is endemic, the possibility of a dengue aetiology in encephalitis should be considered.

\section{References}

1. Annual Health Bulletin, Department of Health, Sri Lanka. 2000, Chapter 5.1, 110.

2. Solomon T, Dung M N, Vaughn D W. Neurological manifestations of dengue infection. Lancet 2000; 355: 1053-8.

3. Sumarmo, Wulur H, Jahja E, Gubler DJ, Sutomenggolo TS, Sulianti Sarosa J. Encephalopathy associated with dengue infection. Lancet 1978; 1: 449-50.

4. Kho L K, Sumarmo, Wulur H, Elly C, Jahja E, Gubler DJ. Dengue haemorrhagic fever accompanied by encephalopathy in Jakarta. Southeast Asian Journal of Tropical Medicine and Public Health 1981; 12: 583-90.

5. Gibbons R V, Vaughn D W. Dengue: an escalating problem. British Medical Journal 2002; 324: 1563-6.

6. Monath TP. Dengue: the risk to developed and developing countries. Proceedings of the National Academy of Science USA 1994; 91: 2395-400.

7. Kuberski TT, Rosen L. A simple technique for the detection of dengue antigen in mosquitoes by immunofluorescence. American Journal of Tropical Medicine and Hygiene 1989; 26: 533-7. 